\title{
An Improvement of Joint Ordering Policy of Multiproduct Inventory Problem with Partial Joint Ordering
}

\author{
Intira Nakunthod \\ Department of Statistics Faculty of Sciences \\ Chiang Mai University, Thailand-50200.
}

\begin{abstract}
This paper discusses an improvement of the joint ordering policy of multiproduct inventory problem with a proposed method, or called as partial joint ordering. The joint ordering policy which is to determine a common ordering interval and all products is requested at the same time, as the proposed partial ordering policy divides products into many clusters. A cluster can be considered as groups of multiproduct being ordered together. Each cluster has its own ordering interval which is independent to each other. The partial joint ordering approach is proposed to perform products clustering and determine the cluster ordering interval. The performance of the proposed partial joint ordering is evaluated at different values of holding cost fraction. A solution which is the average total cost per year yielded from the proposed framework is compared to solutions obtained from the classical individual, joint and mixed ordering policies. The presented partial joint ordering policy performs better than the classical individual, joint and mixed ordering policies with 20.30, 11.30 and 0.87 average cost saving percentages, respectively, from the study-results of 50 generated random problems.
\end{abstract}

Keywords: partial joint ordering, joint ordering policy, multiproduct inventory problem

\section{INTRODUCTION}

The joint ordering policy is a common inventory policy which is dealt with an environment of many products ordered from a single supplier [1] in the same time. The objective is to develop inventory policies so as to minimizing of the total cost per unit time over the infinite horizon [2] and also according to the customer demands. The joint replenishment problem (JRP) is an inventory problem concerning multiple items where economies exist for replenishing several items together [3]. Moreover, the JRP is the problem is occurred in the situations of many products are shared by the same transportation and it may be the situations of packaging of one product after being manufactured in batches [1]. Joint replenishment of a group of items reduces the number of times that the major ordering cost is charged, and so this saves costs [4].

Ordinary, the total cost is composed of two parts which is ordering cost and holding cost [5]. Joint replenishment policy are based on the

interaction of the ordering costs [6].The cost of placing an order to the supplier for a number of different products has two components, that is, a major ordering cost which is independent of the number of products in the order, and a minor ordering cost, which is an individual ordering cost of a particular item is ordered. For some products ordered, the major ordering cost is occurred for using group of ordering may lead to substantial cost savings which are much more significant than the higher major ordering cost [5]. In addition, there is likewise an individual inventory holding cost which is involved to a minor ordering cost. A grouping of several products with having the common order interval and delivering all items at the same time, may have common relationship between the various products in three categories, that is, (a) The holding cost, such as the cost of documents, transportations, inspections, and cargoes, etc., is decreased (b) The unit price of products is decreased, and (c) The customer demand for a product affects to the others. Those relationships may affect to the determination of the optimal order interval of the joint ordering policy and the mixed ordering policy which merges the individual ordering policy and joint ordering policy. Generally, two strategies are presented, which are the algorithms to solve for the joint replenishment problem (JRP) according to direct grouping strategies (DGS) and indirect grouping strategies (IGS). Firstly, DGS is the method which products are divided into predetermined different groups and products within each group are jointly ordered in the same cycle time [1]. Finally, IGS is the method which the placing order is made at regular time intervals and each item has an ordered quantity effective to endure for certainly an integer multiple of those regular time intervals [1].

\section{OBJECTIVE}

The research objective is an improvement of solutions related to the average total costs per year of the multiproduct inventory problem with partial joint ordering policy

\section{SCOPE}

This paper is focused on the study and improvement of solving the multi-items inventory problem with one retailer by means of partial joint delivery many products for improving the average total cost of the inventory system. The scope of this research is composed five articles, that is, (a) The data are simulated by EXCEL program with uniformly random numbers on different closed intervals $[a, b]$. The generated random numbers are used as a representative of customer demands annually, the material cost of each product, major ordering cost, minor ordering cost and holding cost per unit per unit time. (b) Each product is assumed as the classic of 
economic order quantity (EOQ) which is developed by Harris F. [7] but it is also known as the Wilson economic lot size [8] with regardless of the shortage cost. (c) The total cost of the system composes an ordering cost per order of multi-item and the holding cost of each item, with an ignoring of production cost of each item. (d) For each product, customer demands annually, material cost, major ordering cost per order which is independent of the number of items, minor ordering cost which is involved to each item, and the holding cost per unit per unit time are known and constant. (e) All items which are ordered from a vender are delivered to a retailer merchant. (f) The inventory system is considered by multi-item continuous review inventory system [9].

\section{MATERIALS AND METHOD}

\section{MATERIALS}

This paper utilizes three considerable policies which the classical individual, joint and mixed ordering policies for guideline of improvement solution of multiproduct inventory problem with the proposed partial joint ordering policy. For determining the optimal size of lot with the proposed method, the following factors are involved of product 1 of problem $\mathrm{i}$ :

Material cost $\left(c_{i l}\right)$ is the cost in dollars per unit of item 1 under an ordering policy from a supplier.

Major ordering cost $\left(K_{i}\right)$ is the cost of handling an order in an office throughout the factory which is independent of the number of product 1 of problem $i$. This cost which is realized in consideration of this paper, is involved the expenses of handling, checking, indexing, and supervising an order in an office.

Minor ordering cost $\left(k_{i l}\right)$ is an individual ordering cost of product 1 be ordered and it is as well involved an individual inventory holding cost of product 1 .

Holding cost $\left(h_{i l}\right)$ is the cost involved of the carrying inventory of product 1 , because of an interest and depreciation of inventories. The carrying inventory means a lot of money tied up and a heavy depreciation.

Total cost (TC). This is the total cost per unit time of the inventory system problem. It is comprised of the ordering cost and the holding cost. The shortages are not allowed.

Moreover, the following three important theories are involved of the paper. Firstly, the classic individual ordering policy is a method of supplying different products individually. Secondly, the joint ordering policy is a method of providing all items at the same time. Finally, the mixed ordering policy provides multiple different items with combination of the individual ordering and joint ordering. The joint ordering policies for a group of items usually results in a lower total cost, than that resulting from an individual policy for each independently formulated item [8].

\section{THEORITICAL REVIEW}

For determining the proposed method of this paper, the following three policies involved are as follows.

The symbol of $D_{i l}$ and $Q_{i l}$ are used to represent a customer demand per unit time and a quantity of order for product 1 of problem i, respectively.

The individual ordering policy with $\mathrm{m}$ generated problems, $\mathrm{n}$ products of each problem, is stated as,

$T C_{I}=\sum_{i=1}^{m} \sum_{l=1}^{n}\left(c_{i l} D_{i l}+\frac{\left(K_{i}+k_{i l}\right) D_{i l}}{Q_{i l}}+\frac{h_{i l} Q_{i l}}{2}\right)$

where $\mathrm{TC}_{\mathrm{I}}$ denoted by the total cost per unit time of the inventory system of $\mathrm{m}$ problems, $\mathrm{n}$ products of each problem, with the individual ordering policy. For problem i, the optimal value of the whole amount ordered and the order interval of product 1 , with the individual ordering policy, are followed in (2) and (3),

$$
\begin{aligned}
& Q_{i l}=\sqrt{\frac{2\left(K_{i}+k_{i l}\right) D_{i l}}{h_{i l}}} \\
& \mathrm{~T}_{\mathrm{il}}=\sqrt{\frac{2\left(\mathrm{~K}_{\mathrm{i}}+\mathrm{k}_{\mathrm{il}}\right)}{\mathrm{D}_{\mathrm{il}} \mathrm{h}_{\mathrm{il}}}}
\end{aligned}
$$

For the objective of joint ordering policy is given by

$$
\mathrm{TC}_{\mathrm{J}}=\sum_{\mathrm{i}=1}^{\mathrm{m}} \sum_{\mathrm{l}=1}^{\mathrm{n}} \mathrm{c}_{\mathrm{il}} \mathrm{D}_{\mathrm{il}}+\sqrt{2\left(\mathrm{~K}_{\mathrm{i}}+\sum_{\mathrm{l}=1}^{\mathrm{n}} \mathrm{k}_{\mathrm{il}}\right) \sum_{\mathrm{l}=1}^{\mathrm{n}} \mathrm{D}_{\mathrm{il}} \mathrm{h}_{\mathrm{il}}}
$$

where $\mathrm{TC}_{\mathrm{J}}$ denoted by the total cost per unit time of the inventory system of $\mathrm{m}$ problems, $\mathrm{n}$ products for each problem, with the joint ordering policy.

For problem i, the basic order interval for replenishing all $n$ products together and the optimal lot size of product 1 , with the joint ordering policy, respectively, can be

$$
\begin{aligned}
& \mathrm{T}_{\mathrm{i}}=\sqrt{\frac{2\left(\mathrm{~K}_{\mathrm{i}}+\sum_{\mathrm{l}=1}^{\mathrm{n}} \mathrm{k}_{\mathrm{il}}\right)}{\sum_{\mathrm{l}=1}^{\mathrm{n}} \mathrm{D}_{\mathrm{il}} \mathrm{h}_{\mathrm{il}}}} \\
& Q_{i l}=D_{i l} T_{i}
\end{aligned}
$$

Therefore, $\mathrm{TC}_{\mathrm{M}}$ denoted by the total cost per unit time of the inventory system with the mixed ordering policy be composed of $\mathrm{m}$ problems, $\mathrm{n}$ products of each problem, indicates

$$
\begin{aligned}
T C_{M}=\sum_{i=1}^{m} \sum_{l=1}^{n} c_{i l} D_{i l} & \\
& +\sqrt{2\left(K_{i}+\sum_{l=1}^{n} \frac{k_{i l}}{\left\lceil\alpha_{i l}\right\rceil}\right) \sum_{l=1}^{n} h_{i l} D_{i l}\left\lceil\alpha_{i l}\right\rceil(7)}
\end{aligned}
$$

For problem i, the relative order interval of product 1 , which be thus the temporary interval time for establishing relative 
ordering frequency by the mixed ordering policy satisfies

$\tau_{i l}=\sqrt{\frac{2 k_{i l}}{D_{i l} h_{i l}}}$

The ascending order of the relative order interval of problem $\mathrm{i}$ is performed, we get

$\tau_{\mathrm{i} 1} \leq \tau_{\mathrm{i} 2} \leq \tau_{\mathrm{i} 3} \leq \cdots \leq \tau_{\mathrm{in}}$

The value of $\tau_{\mathrm{i} 1}$ and $\tau_{\mathrm{in}}$ signify the minimum and maximum relative order interval, respectively. For product 1 of problem $\mathrm{i}$, the relation of the value of $\tau_{i l}^{\prime}$ and $\tau_{i 1}^{\prime}$ demonstrates

$\tau_{i l}=\alpha_{i l} \tau_{i 1}$

where $1 \leq \alpha_{i 1} \leq \alpha_{i 2} \leq \cdots \leq \alpha_{i n}$. For each value of $\alpha_{i j}$, which be the real number, means the ratio of $\tau_{i l}$ and $\tau_{i 1}$. Let $\alpha_{i l}=1$ for any $k_{i l}=0$ [8]. Therefore, the absolute order interval for product 1 of problem i can be satisfied by

$\mathrm{T}_{\mathrm{il}}=\left\lceil\alpha_{i l}\right\rceil T_{i}$

The value of $\left\lceil\alpha_{i j}\right\rceil$ is the nearest integer number of $\alpha_{i j}$ and $T_{i 1}$ is the smallest absolute order interval which be occurred during the replenishment of multiple different products for problem i can be

$\mathrm{T}_{\mathrm{i} 1}=\sqrt{\frac{2\left(\mathrm{~K}_{\mathrm{i}}+\sum_{\mathrm{l}=1}^{\mathrm{n}} \frac{\mathrm{k}_{\mathrm{il}}}{1\left[\alpha_{\mathrm{il}}\right]}\right)}{\sum_{\mathrm{l}=1}^{\mathrm{n}} \mathrm{D}_{\mathrm{il}} \mathrm{h}_{\mathrm{il}}\left\lceil\alpha_{\mathrm{il}}\right\rceil}}$

Thus, the optimal lot size of product 1 of problem i can be demonstrated by

$Q_{i l}=D_{i l}\left\lceil\alpha_{i l}\right\rceil T_{i l}$

\section{Data}

The data of this paper were simulated by random numbers with uniform distribution on different closed intervals $[a, b]$ of fifty problems, five products for each problem. For item 1 of problem $i$, the uniform random numbers are generated for customer demand rate per year, material cost per unit, major ordering cost per order, minor ordering cost per unit and holding cost per unit per year. The simulated data of this paper, for item 1 of problem $i$, is given below:

(a) Customer demand per year of item 1 defined by $D_{i l}$ is a random number on closed interval [1000, 5000].

(b) Material cost of item 1 defined by $c_{i l}$ is a random number on closed interval $[10,50]$. (c) Major ordering cost per order of problem i defined by $\mathrm{K}_{i}$ is a random number on closed interval [1000, 5000].

(d) Minor ordering cost for item 1 defined by $\mathrm{k}_{i l}$ is on closed interval $[0.5,2.0]$ which is proportional to a major ordering cost per order. It is given three values, that is, 0.5, 1.0 and 1.5, respectively.

(e) Holding cost per unit per year of item 1 defined by $h_{i l}$ is a given percentage of material cost, that is, $10 \%$ of material cost.

\section{METHOD}

The proposed method called partial joint ordering is used to solve the joint ordering problem with multiple different products for one retailer. This method is used to classify items into different groups in each problem. The significant condition of products clustering is the joint ordering is better than the classical individual ordering with corresponding to the critical inequality in (13) as follows.

For problem i with item $\mathrm{u} \neq \mathrm{v}$;

$2\left(K_{i}+k_{i u}+k_{i v}\right)\left(h_{i u} D_{i u}+h_{i v} D_{i v}\right)$

$\leq \sqrt{2\left(K_{i}+k_{i u}\right) h_{i u} D_{i u}}+\sqrt{2\left(K_{i}+k_{i v}\right) h_{i v} D_{i v}}$

Here, inequality (13) is used for comparing two items per time. The proposed heuristic, for solving fifty problems, is clustering items with the basic individual time interval of each item by the classical individual ordering policy. These ordering time intervals are sorted in ascending order. The presented algorithm is used to solve each problem with four steps as follows:

Step 1: For item 1 of problem i, random numbers with uniform distribution are generated for customer demand $D_{i l}$, material cost per unit $c_{i l}$, major ordering cost per order $K_{i}$, minor ordering cost per unit $k_{i l}$ and holding cost per unit per year $h_{i l}$.

Step 2: For item 1 of problem i, the order interval is calculated by the classical individual ordering and five items of each problem are sorted in ascending order.

Step 3: Each grouping is done with two items by using (13). All five items in each problem are used to check in (13) entirely as followed:

-If it is true, then a group will be done by joint ordering because the total cost per year of joint ordering is lower than the total cost per year of individual ordering.

- if it is not true, then a group will not be done because the total cost per year of individual ordering policy is lower than the total cost per year of joint ordering policy.

Step 4: All groups of products of each problem, which each group has its own order interval, are occurred by the previous 
step 3, and used to calculate the total cost per year of mixed ordering policy.

By this means, the total cost per year of each problem with different major ordering cost, and minor ordering cost and holding cost fraction of each item is calculated. The popular average cost savings of a partial joint ordering policy, which is a dimensionless term and is denoted by $y_{P}$, can be expressed as a percentage of the total cost per year of an independent policy as follows in [14]:

$y_{P}=100 \cdot \frac{T C_{T}-T C_{P}}{T C_{T}}$

where $T C_{T}$ denoted the total cost per year of the individual, joint and mixed ordering policy, and $T C_{P}$ is the total cost per year of the partial joint ordering policy.

\section{RESULTS AND DISCUSSION}

This paper considers the following case study fifty problems which each problem has five products. The algorithm of improvement is used to solve the example of problem $i$ as the following of four steps.

Step 1: Random numbers are generated with a uniform distribution for annual customer demand $D_{i l}$, material cost per unit in dollars $\mathrm{c}_{i l}$, major ordering cost in dollars $K_{i}$, as the calculating of each item of minor ordering cost $k_{i l}$ and holding cost $h_{i l}$ are occurred in dollars.

Step 2: By the classical individual ordering policy, the order interval of each item in years $T_{i l}$ calculated by equation (3) is sorted in ascending order and summarized in Table 1:

Table 1: Order intervals of individual ordering and ordinal number of products of example

\begin{tabular}{|c|c|c|c|c|c|c|}
\hline \multicolumn{7}{|c|}{ Major ordering cost $\left(K_{i}\right)=2,864.8$} \\
\hline $\begin{array}{c}\text { Number } \\
\text { of } \\
\text { product 1 }\end{array}$ & $D_{i l}$ & $c_{i l}$ & $\mathrm{k}_{i l}$ & $h_{i l}$ & $T_{i l}$ & $\begin{array}{c}\text { Ordinal } \\
\text { number of } \\
\text { products }\end{array}$ \\
\hline 1 & 3521 & 23.0261 & 2422 & 2.3026 & 1.1420 & $\mathbf{2}$ \\
\hline 2 & 3413 & 33.1954 & 4010.2 & 3.3195 & 1.1016 & $\mathbf{1}$ \\
\hline 3 & 1142 & 27.9371 & 3640.4 & 2.7937 & 2.0194 & $\mathbf{5}$ \\
\hline 4 & 1432 & 26.3092 & 3408.6 & 2.6309 & 1.8249 & $\mathbf{4}$ \\
\hline 5 & 3205 & 28.0353 & 4893.4 & 2.8035 & 1.3141 & $\mathbf{3}$ \\
\hline
\end{tabular}

Step 3: The grouping of two items is used by the inequality (13) with the following steps.

3.1 By consideration of the ordinal number of products in Table 1, the first and the second order interval of product number are determined to be possibly grouped together. Therefore, the order interval of products $\{2\}$ and $\{1\}$, grouped together to become possible three clusters, which are $\{2,1\}$, $\{2\}$ and $\{1\}$. The following steps of calculation are performed as follows:

a) By the joint ordering policy, the total cost per year of group $\{2,1\}$, with the ordering cost being $\$ 9,297$ is equal to $\$ 19,011$.

b) By the individual ordering policy, the total cost per year of a combination of two products, $\{2\}$ and $\{1\}$, with the ordering cost be $\$ 5,286.8$ and $\$ 6,875$, respectively, is valued at $\$ 21,739.96$.

c) By comparing the total cost per year of group $\{2,1\}$ to the total cost per year of a combination of the two products, $\{2\}$ and $\{1\}$, with inequality (13), the total cost per year of group $\{2,1\}$ is lower than the total cost per year of a combination of two products, $\{2\}$ and $\{1\}$. Hence, a grouping of products $\{2,1\}$ is discovered.

3.2 By consideration of the ordinal number of products in Table 1 , the order interval of grouping $\{2,1\}$ and the third order interval of product number, that is $\{5\}$, are determined to be possibly grouped together. The possible three groups of order interval are occurred, which are $\{2,1,5\},\{2,1\}$ and $\{5\}$. The following steps of calculation are performed as follows:

a) By the joint ordering policy, the total cost per year of group $\{2,1,5\}$ with the ordering cost be $\$ 14,190.4$, is equal to $\$ 36,897.08$.

b) By the individual ordering policy, the total cost per year of a combination of two product, $\{2,1\}$ and $\{5\}$, with the ordering cost be $\$ 9,297$ and $\$ 7,758.2$, respectively, is equal to $\$ 30,818.33$.

c) By comparing of the total cost per year of group $\{2$, $1,5\}$ to the total cost per year of a combination of two groups, $\{2,1\}$ and $\{5\}$, with inequality (13), the total cost per year of group $\{2,1,5\}$ is more than the total cost per year of a combination of two groups, $\{2,1\}$ and $\{5\}$. Hence, two grouping of products $\{2$, $1\}$ and $\{5\}$ are found out.

3.3 By consideration of the fourth order interval of product number $\{5\}$ and $\{4\}$ are determined to be possibly grouped together. The possible three groups of order interval are occurred which being $\{5,4\},\{5\}$ and $\{4\}$. The following steps are calculated as follows:

a) By the joint ordering policy, the total cost per year of group $\{5,4\}$, with the ordering cost be $\$ 11,166.8$, is equal to $\$ 16,876$.

b) By the individual ordering policy, the total cost per year of a combination of two products, $\{5\}$ and $\{4\}$, with the ordering cost be $\$ 7,758.2$ and $\$ 6,273.4$, respectively, is equal to $\$ 18,682.83$.

c) By comparing the total cost per year of group $\{5,4\}$ to the total cost per year of a combination of two groups, $\{5\}$ and $\{4\}$, with inequality (13), the total cost per year of group $\{5,4\}$ is lower than the total cost per year of a combination of two groups, $\{5\}$ and $\{4\}$. Hence, we get a group of products $\{5,4\}$. 
3.4 By consideration of the fifth order interval of product number in Table 1 , which being $\{3\}$, the order interval of grouping of product number $\{2,1\}$ and $\{3\}$ are considered to be possibly grouped together. The possible three clusters of order interval are occurred which being $\{2,1,3\},\{2,1\}$ and $\{3\}$. The following steps are calculated as follows:

a) By the joint ordering policy, the total cost per year of group $\{2,1,3\}$ with the ordering cost be $\$ 12,937.4$, is equal to $\$ 33,034$.

b) By the individual ordering policy, the total cost per year of a combination of two groups, $\{2,1\}$ and $\{3\}$, with the ordering cost be $\$ 9,297$ and $\$ 6,505.2$, respectively, is equal to $\$ 25,453$.

c) By comparing the total cost per year of group $\{2,1$, $3\}$ to the total cost per year of a combination of two product groups, $\{2,1\}$ and $\{3\}$, with inequality (13), the total cost per year of group $\{2,1,3\}$ is more than the total cost per year of a combination of two groups, $\{2,1\}$ and $\{3\}$. Hence, two groups of products $\{2,1\}$ and $\{3\}$ are discovered.

3.5 Consider the fifth order interval of product number in Table 1, which being $\{3\}$, the order interval of grouping of product number $\{5,4\}$ and $\{3\}$ are considered to be possibly grouped together. The three clusters are in the forms of $\{5,4$, $3\},\{5,4\}$ and $\{3\}$. The following steps of calculation are performed as follows:

a) By the joint ordering policy, the total cost per year of group $\{5,4,3\}$ with the ordering cost be $\$ 11,166.8$, is equal to $\$ 25,180.24$.

b) By the individual ordering policy, the total cost per year of a combination of two groups of products, $\{5$, $4\}$ and $\{3\}$, with the ordering cost be $\$ 11,166.8$ and $\$ 6,505.2$, respectively, is equal to $\$ 23,319.11$.

c) By Comparing the total cost per year of group $\{5,4$, $3\}$ to the total cost per year of a combination of two groups, $\{5,4\}$ and $\{3\}$ with inequality (13), the total cost per year of group $\{5,4,3\}$ is more than the total cost per year of a combination of two groups, $\{5,4\}$ and $\{3\}$. Hence, two groups of item $\{5,4\}$ and $\{3\}$.

Frequently, we get finally grouping of products $\{5,4\}$ and $\{3\}$ are discovered.

Finally, we get three groups of products $\{2,1\},\{5,4\}$ and $\{3\}$ for this problem by step 3.1 thorough 3.5 .

Step 4: By the mixed ordering policy, the relative order interval of grouping $\{2,1\},\{5,4\},\{3\}$ are valued at 0.9781 , 1.3234 and 2.1094 years, respectively. The smallest order interval with equation (12) is valued at 1.0035 years and the total cost per year of these groups is valued at $\$ 38,704.16$.

From the previous following steps of calculation, all five products are also provided with the same multiple of 1.0035 years as the multiple of times of absolute order intervals are shown in the form of any even integer. It means that a group of product number $\{2,1,5,4\}$ is ordered for every period of 1.0035 years, while a group of product number $\{2,1,5,4,3\}$ is ordered for every period of 2.0069 years. Therefore, the total cost of partial joint ordering policy of this problem and the final results of fifty problems can be summarized in Table 2, and Table 3 as follows.

Table 2: The total cost per year of example problem with Individual, joint, mixed and partial joint ordering policies

\begin{tabular}{lcccc}
\hline \hline Problem & Individual & Joint & Mixed & Partial joint \\
\hline \hline 1 & $46,865.50$ & $38,767.24$ & $38,730.10$ & $38,704.16$ \\
2 & $45,426.68$ & $41,177.23$ & $38,831.57$ & $39,323.14$ \\
3 & $59,316.19$ & $49,184.99$ & $50,154.05$ & $46,801.07$ \\
4 & $69,202.47$ & $58,296.60$ & $58,810.35$ & $58,810.35$ \\
5 & $55,971.19$ & $45,742.41$ & $46,767.57$ & $44,514.78$ \\
& $\vdots$ & $\vdots$ & $\vdots$ & $\vdots$ \\
50 & $40,785.54$ & $32,295.28$ & $32,733.44$ & $32,733.44$ \\
\hline \hline
\end{tabular}

Table 3: The final results of fifty generated problems

\begin{tabular}{lrccc}
\hline \hline $\begin{array}{l}\text { Time } \\
\text { delivery }\end{array}$ & $\begin{array}{r}\text { of Period } \\
\text { (years) }\end{array}$ & $\begin{array}{r}\text { of } \\
\text { shipped }\end{array}$ & $\begin{array}{r}\text { delivery List of items } \\
\text { no. }\end{array}$ \\
\hline \hline 1 & 1.0035 & $2,1,5,4$ & \\
2 & 2.0069 & $2,1,5,4,3$ & \\
3 & 3.0104 & $2,1,5,4$ & \\
4 & 4.0139 & $2,1,5,4,3$ & \\
5 & 5.0173 & $2,1,5,4$ & $\vdots$ \\
& & $\vdots$ & & \\
\hline \hline
\end{tabular}

\section{CONCLUSIONS}

The proposed method or the partial joint ordering policy can be concluded that the percentages of an average cost saving with equation (14) are 20.30, 1.13 and 0.87 , respect to the classical individual, joint and mixed ordering policies respectively. In this paper, we propose various holding cost fractions for improving solutions of multiproduct inventory problems with the partial joint ordering policy. The developers can provide customized rules for grouping multiproduct. Moreover, the reader can add new rules and heuristics for grouping of items jauntily.

\section{REFERENCES}

[1] Nilsson A, Segerstedt A, van der Sluis E.A new iterative heuristic to solve the joint replenishment problem using a spreadsheet technique. International Journal Economic Production, 2007; 108:399-405.

[2] Viswanathan S.A new optimal algorithm for the joint replenishment problem. Journal of the Operational Research Society, 2002; 53:1286-90.

[3] Viswanathan S. On optimal algorithms for the joint 
replenishment problem. Journal of the Operational Research Society, 1996; 47:936-44.

[4] Wildeman RE, Frenk JBG, Dekker R. An efficient optimal solution method for the joint replenishment problem. European Journal of Operational Research, 1997; 99:433-44.

[5] Khouja M, Goyal S. A review of the joint replenishment problem literature: 1989-2005. European Journal of Operational Research, 2008; 186:1-16.

[6] van Eijs MJG, Heuts RMJ, Kleijnen JPC. Analysis and comparison of two strategies for multi-item inventory systems with joint replenishment costs. European Journal of Operational Research, 1992; 59:405-12.

[7] Harris F. Operations and Cost (Factory Management Series). Chicago: A.W. Shaw Company; 1995.

[8] Love SF. Inventory Control. $1^{\text {st }}$ ed. Tokyo: McGrawHill; 1979.

[9] Lee CY, Lee D. An efficient method for solving a correlated multi-item inventory. Operation Research Perspectives, 2018; 5:13-21. Available online: 2 December 2017. 